\title{
Incompatibility between Einstein's general relativity and Heisenberg's uncertainty principle
}

\author{
Alexandre Georges ${ }^{\text {a) }}$ \\ 51 Redcliffe Street, Keighley, West Yorkshire BD21 2PX, United Kingdom
}

(Received 6 May 2018; accepted 18 June 2018; published online 12 July 2018)

\begin{abstract}
Are General Relativity and Quantum Mechanics incompatible? Each in their world, that of the infinitely large and that of the infinitely small, they did not seem to interfere as long as they avoided each other. However, it is their fundamental oppositions that prevent the scientific community from achieving a unification of physics. The proposal of this paper is to provide a mathematical proof of incompatibility, beyond the fact that they have fundamentally different principles, between the foundations of General Relativity and Quantum Mechanics, namely, the deformation of the space-time geometry and the Uncertainty Principle. It will thus be possible to provide an absolute limitation in establishing a unifying theory of physics, if any. Moreover, while respecting the conditions fixed by the Uncertainty Principle, it will be tempted to determine with accuracy and simultaneity, the position and the speed of a nonrelativistic particle, by application of relativistic principles and bypassing the problems raised by such an operation. The Uncertainty Principle as stated by Werner Heisenberg will be then, in the light of observations made on the measurement of the time dilatation and in accordance with its own terms, refuted by the present. @ 2018 Physics Essays Publication. [http://dx.doi.org/10.4006/0836-1398-31.3.327]
\end{abstract}

Résumé: La Relativité Générale et la Mécanique Quantique sont-elles incompatibles? Chacune dans son monde, celui de l'infiniment grand et celui de l'infiniment petit, elles ne semblent pas interférer tant qu'elles s'évitent. Cependant, ce sont leurs oppositions fondamentales qui empêchent la communauté scientifique de réaliser une unification de la Physique. La proposition de cet article est de fournir une démonstration mathématique d'incompatibilité, au-delà du simple fait qu'elles aient des principes fondamentalement différents, entre les piliers de la Relativité Générale et de la Mécanique Quantique, soient la déformation de la géométrie de l'espace-temps et le Principe d'Incertitude. Il sera ainsi possible de définir une limitation absolue à l'établissement d'une théorie unificatrice de la Physique, s'il en est. De plus, tout en respectant les conditions fixées par le Principe d'Incertitude, il sera tenté de déterminer avec précision et simultanéité la position et la vitesse d'une particule non relativiste, en appliquant des principes relativistes tout en contournant les problèmes posés par une telle opération. Cette incompatibilité pourrait indiquer que la dilatation du temps, le Principe d'Incertitude ou encore notre vision d'une unification potentielle de la Physique pourraient être révisées. Le Principe d'Incertitude énoncé par Werner Heisenberg sera ainsi, au regard d'observations et mesures effectuées sur la dilatation du temps et conformément à ses propres conditions, réfuté par le présent développement.

Key words: Time; General Relativity; Uncertainty Principle; Quantum Mechanics; Unification of Physics; Photon; Particle.

\section{INTRODUCTION}

General Relativity and Quantum Mechanics are two ways of conceiving the nature of the Universe. On the one hand, gravity is described as the result of the deformation of the geometry of Space-Time. The nature of the universe is here deterministic. On the other hand, we describe discontinuous forces between subatomic particles whose leaders are bosons. Randomness is intrinsic to the nature of this Universe. Both models usually apply to different scales and situations.

It will be tempted to demonstrate the incompatibility between the fundamental principles of the two models: General Relativity and Quantum Mechanics, respectively, the time dilatation principle and the Uncertainty Principle.

${ }^{a)}$ professor.alexandre.georges@gmail.com
It will be demonstrated here that, first, the conservation of energy is relative and that, second, we can precisely and simultaneously determine the momentum and the position of a subatomic particle, bypassing the problems related to the nonrelativistic aspect of said particle. We will thus demonstrate, by two extremely simple mathematical proofs, the incompatibility between the time dilatation principle and the Heisenberg's Uncertainty Principle, before interpreting the results on the basis of observations made on the measurement of the time dilatation.

\section{THE RELATIVE CONSERVATION OF ENERGY}

Let $\Omega$ be an object of mass $M$ and $\Phi$ a photon of frequency $\nu$ and energy $E$.

In the first situation, $\Phi$ is at a distance $r_{1}$ of $\Omega$.

In the second situation, $\Phi$ is at a distance $r_{2}$ of $\Omega$. 
In both situations, $\Phi$ is observed from an almost flat space-time.

In first situation, the proper time $d \tau_{1}$, relative to the point in which $\Phi$ is located in the first situation, is expressed by

$$
d \tau_{1}=d t \sqrt{1-\frac{\frac{2 G M}{c^{2}}}{r_{1}}} .
$$

In second situation, the proper time $d \tau_{2}$, relative to the point in which $\Phi$ is located in the second situation, is expressed by

$$
d \tau_{2}=d t \sqrt{1-\frac{\frac{2 G M}{c^{2}}}{r_{2}}} .
$$

If

$$
r_{1}<r_{2}
$$

then

$$
d \tau_{1}<d \tau_{2}
$$

Thus, the more $\Phi$ moves away from $\Omega$, the more the time $d \tau$ proper to the point where the photon $\Phi$ is, from the point of view of an observer situated in an almost flat spacetime, increases with respect to $d t$.

But

$$
E=h \times \nu .
$$

We will note $E_{1}$ the energy of $\Phi$ in first situation and $E_{2}$ the energy of $\Phi$ in second situation.

So, if we apply the time dilatation to the frequency of the photon wave, we have in first situation

$$
E_{1}=\frac{h}{d t \sqrt{1-\frac{\frac{2 G M}{c^{2}}}{r_{1}}}}
$$

then

$$
E_{1}=\frac{h}{d \tau_{1}}
$$

and in second situation

$$
E_{2}=\frac{h}{d t \sqrt{1-\frac{\frac{2 G M}{c^{2}}}{r_{2}}}}
$$

then

$$
E_{2}=\frac{h}{d \tau_{2}} .
$$

But

$$
d \tau_{1}<d \tau_{2}
$$

so

$$
E_{1}>E_{2}
$$

The proper times $d \tau_{1}$ and $d \tau_{2}$, derived from the Schwarzschild metric, ${ }^{1}$ are to be differentiated from the proper time of the photon $\Phi$, these, respectively, corresponding to the times proper to the positions of the photon $\Phi$ in the first and second situations, while $d \tau$ corresponds to the time proper to the photon itself, as a particle.

Finally, from the point of view of an observer situated in an almost flat space-time, the more $\Phi$ moves away from $\Omega$, the lower its energy level decreases, without any energy being transferred from $\Phi$ to its environment. Also, the closer $\Phi$ gets to $\Omega$, the more its energy increases, again from the point of view of an observer situated in an almost flat space-time, without any energy being brought to the system that $\Phi$ constitutes.

In conclusion, from the point of view of an observer situated in an almost flat space-time, the energy level of a photon depends on its frequency, depending on the deformation of the geometry of the space-time in which it is located. The conservation of the energy is then relative.

\section{APPLICATION OF THE RELATIVE CONSERVATION OF ENERGY TO A SUBATOMIC PARTICLE'S SYSTEM SUBJECT TO THE HEISENBERG'S UNCERTAINTY PRINCIPLE}

Let us start by considering that

$$
\sigma_{x} . \sigma_{p} \geq \frac{\hbar}{2}
$$

where

$$
\hbar=\frac{h}{2 \pi} \text {. }
$$

It is, according to the Uncertainty Principle and as expressed through its fundamental equation, essentially impossible to determine with precision both the position $x$ and the momentum $p=m v$ (and therefore by extension the velocity) of a particle, regardless of observational qualities. $^{2-4} \mathrm{We}$ apply this theorem (the principle being demonstrable, especially using Fourier transformation) to nonrelativistic particles. So, we will avoid, in this section, to directly apply relativistic principles to the particle, but we will consider the deformation of the space-time geometry to the point where the particle is located.

\section{A. Application}

As seen in Section II, the energy of a photon can vary according to its position. It is then possible, for an observer in an almost flat space-time, to determine the position of the particle moving on an axis, knowing its level of energy. 
Imagine that we are this observer. Our particle $\Phi$, of known mass $m$, moves toward another object $\Omega$ of known mass $M . M$ is much higher than $m$. The object $\Omega$ is immobile, without rotation, and homogeneous. The axis corresponding to the trajectory of the particle $\Phi$ is known and rectilinear. Our object $\Omega$ is very far, in our simple example, from any other mass object than our particle $\Phi$. As our $\Phi$ moves toward our isolated $\Omega$ of mass $M$, it never has the same level of energy from one position to another.

If we are able to determine with precision the momentum and the position of the particle, precisely and simultaneously, by applying relativistic principle on the point in which our particle is located, we will prove incompatibility between General Relativity and Quantum Mechanics.

Moreover, the fact that the impossibility of determination, namely, uncertainty, is fundamental and independent of the observational qualities does not make the improbability of the previously proposed observational method responsible for the simultaneous and precise determination of the momentum and the position of the particle.

In view of De Broglie's thesis, generalizing the Einstein-Planck relation by applying it to mass particles, we can express

$$
\begin{aligned}
& \lambda=\frac{h}{p}, \\
& \lambda=\frac{h}{m v} .
\end{aligned}
$$

As seen by the principles of pair production process and annihilation process, ${ }^{5}$ the mass of our particle corresponds to a potential amount of energy that can be produced. We will then study the particle as a quantity (or more generally a set of quantities) of energy. Thus, if we cannot use the dilatation of the time undergone by a nonrelativistic particle, we can determine, however, as seen in Section II, the relative energy level corresponding to the mass of the particle, considering the photon that it could be (or more generally the set of quantities of masses that could be removed in the form of extractable energy). Thus, from the quantity (here set of quantities) of mass, we would consider the quantity (here set of quantities) of relative energy producible from it. All this could make it possible to circumvent the problem of the nonrelativistic aspect of the studied particle, the relative conservation of the energy being applied to the quanta of energy.

Considering that $\Phi$ is a particle with a mass from which quantity of energy, quantum by quantum, can be withdrawn up to a finite energy quantity $e$, corresponding to $m c^{2}$, we will express $m$ by its set of masses

$$
m=\sum_{i=1}^{i \rightarrow n} m_{i}=m_{1}+m_{2}+m_{3}+\ldots+m_{n},
$$

where $n$ is a finite positive integer and where each $m_{i}$ is a part of the particle mass $m$.

Then

$$
\sum_{i=1}^{i \rightarrow n} m_{i}=\sum_{i=1}^{i \rightarrow n} \frac{E}{c^{2}}
$$

Also

$$
\frac{E}{c^{2}}=\sum_{i=1}^{i \rightarrow n} \frac{E_{i}}{c^{2}}=\frac{E_{1}}{c^{2}}+\frac{E_{2}}{c^{2}}+\frac{E_{3}}{c^{2}}+\ldots+\frac{E_{n}}{c^{2}},
$$

where each $E_{i}$ is a quantum of extractable energy.

So

$E=\sum_{i=1}^{i \rightarrow n} E_{i}$

Considering the proof set out in Section II of this file

$E=\frac{h}{d \tau}$.

We can express

$$
\sum_{i=1}^{i \rightarrow n} E_{i}=\sum_{i=1}^{i \rightarrow n} \frac{h}{d \tau_{i}}
$$

and

$$
\sum_{i=1}^{i \rightarrow n} \frac{h}{d \tau_{i}}=\frac{h}{d \tau}
$$

to finish by

$$
\mu=\frac{E}{c^{2}}=\frac{h}{c^{2} d \tau} .
$$

The mass $\mu$ is here considered as the mass corresponding to the potentially extractable energy, from the point of view of an observer located in an almost flat space-time. The proof provided in Section II covers the case of a quantum of energy, the photon. The mass of the particle does not vary, from the point of view of an observer situated in an almost flat spacetime, according to the curvature of the space-time geometry, considering the nonrelativistic aspect of the particle required by the Uncertainty Principle and the fact that the proof provided in Section II concerns quanta of energy. But the energy level, from the point of view of an observer situated in an almost flat space-time, varies according to the curvature of the space-time geometry, given the fact that its energy $E$ is relative to the frequency $\nu$ of its wave. The mass $\mu$ is, therefore, not the exact mass $m$ of the particle, but the mass corresponding to the extractable relative energy level $E$.

$$
\begin{aligned}
& \text { If } \\
& e=\frac{h}{d t}
\end{aligned}
$$

and

$$
E=\frac{h}{d t \sqrt{1-\frac{\frac{2 G M}{c^{2}}}{r}}},
$$


we can say, that

$$
E=\frac{e}{\sqrt{1-\frac{\frac{2 G M}{c^{2}}}{r}}}
$$

so

$$
e=E \sqrt{1-\frac{\frac{2 G M}{c^{2}}}{r}}
$$

and

$$
m=\frac{E \sqrt{1-\frac{\frac{2 G M}{c^{2}}}{r}}}{c^{2}}
$$

Now, as expressed here

$$
\lambda=\frac{h}{m v} .
$$

If we know $v$ and $m$, we know $\lambda$.

As stated above, considering the nonrelativistic aspect of the particle (a subatomic particle with a positive mass $m$ ), we cannot directly assign a relative mass $\mu$ to our particle, given the fact that the $m$ mass of our particle does not vary depending on the curvature of the space-time geometry. However, the releasable energy $e$ has no physical existence either, from the point of view of an observer located in an almost flat space-time. Thus, from the point of view of this observer, the totality of the releasable energy that he could perceive, given the fact that he is not limited as regards observational capacity, is the extractable relative energy $E$. The said observer cannot determine observatively $\mu$, since it does not have a demonstrable physical existence, but he can consider $E$, without knowing the position of the particle, by establishing it by the observation, as being the extractable energy $e$ (or here the set of extractable energy quantities) modified by the deformation of the space-time geometry that this potential value naturally undergoes, as stated in Section II. To conclude, our observer, being not limited as regards observational capacity, cannot know the relative mass $\mu$ of the particle, because of its physical inexistence. But he can know the set of extractable energy quantities $E$, whose energy level varies according to the deformation of the geometry of space-time, as a parameter of the system of the particle, if we naturally consider its physical existence as shown above.

Nota bene: It may be difficult to conceive that the observer can determine $E$ by observation. But the Uncertainty Principle must work independently of the available observation capabilities. Thus, our observer can potentially observe everything and access any data relative to the particle by observation. The only constraint is that it cannot determine by the observation with precision and simultaneity the position $x$ of our particle $\Phi$, if it already knows precisely the speed $v$ (or $p$ or $\lambda$ ). Even if the question is not closed, we will first consider the physical existence of the $E$ parameter of $\Phi$.

\section{B. Development}

Now that we know $v$, we know $\lambda$, and nothing in the Uncertainty Principle prevents us from observing by observation the set $E$ of extractable relative energy quantities of our particle at the same time.

Thus, we can develop

$$
\begin{aligned}
\lambda= & \frac{h}{m v}, \\
\lambda= & \frac{\frac{h}{\sqrt{1-\frac{2 G M}{c^{2}}}}}{c^{2}} v
\end{aligned}
$$

$\frac{E \sqrt{1-\frac{\frac{2 G M}{c^{2}}}{r}}}{c^{2}}=\frac{h}{\lambda v}$,

$E \sqrt{1-\frac{\frac{2 G M}{c^{2}}}{r}}=\frac{h c^{2}}{\lambda v}$,

$\sqrt{1-\frac{\frac{2 G M}{c^{2}}}{r}}=\frac{h c^{2}}{\lambda v E}$,

$1-\frac{\frac{2 G M}{c^{2}}}{r}=\left(\frac{h c^{2}}{\lambda v E}\right)^{2}$,

$\frac{\frac{2 G M}{c^{2}}}{r}=1-\left(\frac{h c^{2}}{\lambda v E}\right)^{2}$,

$\frac{2 G M}{c^{2}}=\left(1-\left(\frac{h c^{2}}{\lambda v E}\right)^{2}\right) r$,

$$
\frac{\frac{2 G M}{c^{2}}}{1-\left(\frac{h c^{2}}{\lambda v E}\right)^{2}}=r,
$$

where $r$ is the distance between $\Omega$ and $\Phi$, our particle moving toward the object $\Omega$ of known mass $M$.

Finally, knowing by observation $E, v$, and $\lambda$, we know $r$, so the position $x$ of $\Phi$ on the axis, as an observer with no limitations on observational capability.

We can say, that it is entirely possible, according to General Relativity, to determine, with precision and simultaneity, the momentum and the position of the same particle. 


\section{DIRECT APPLICATION TO THE WAVE PERIOD OF A SUBATOMIC PARTICLE SUBJECT TO THE HEISENBERG'S UNCERTAINTY PRINCIPLE}

The possibility of determining the extractible relative energy, as seen in Section III, could be questionable. Let us try again to determine the position of the object, this time without having to observe $E$. We will use the dilatation of the wave period of the particle, as seen in Section II.

We observe again the speed $v$ of our particle $\Phi$, of known mass $m$, moving on a known axis, toward the object $\Omega$ of mass $M$.

However, we know that

$$
v=\frac{\lambda}{\theta} \text {. }
$$

So

$$
v \theta=\frac{h}{m v}
$$

and

$$
\theta=\frac{h}{m v^{2}},
$$

where $\theta$ is the wave period.

We observe now, as an observer located in an almost flat space-time, not limited in its observation capacities, the relative period $\Theta$ of the wave, corresponding to the $\theta$ period dilated by the gravitational field of $\Omega$. Indeed, the Uncertainty Principle does not preclude the precise and simultaneous determination of the speed and the relative period of the wave of the particle. We will express it

$$
\begin{aligned}
& \Theta=\theta \sqrt{1-\frac{\frac{2 G M}{c^{2}}}{r}}, \\
& \theta=\frac{\Theta}{\sqrt{1-\frac{\frac{2 G M}{c^{2}}}{r}}},
\end{aligned}
$$

and finally develop

$$
\begin{aligned}
& \theta=\frac{h}{m v^{2}}, \\
& \frac{\Theta}{\sqrt{1-\frac{\frac{2 G M}{c^{2}}}{r}}}=\frac{h}{m v^{2}}, \\
& \sqrt{1-\frac{\frac{2 G M}{c^{2}}}{r}}=\frac{m v^{2} \Theta}{h},
\end{aligned}
$$

$$
\begin{aligned}
& 1-\frac{\frac{2 G M}{c^{2}}}{r}=\left(\frac{m v^{2} \Theta}{h}\right)^{2}, \\
& \frac{\frac{2 G M}{c^{2}}}{r}=1-\left(\frac{m v^{2} \Theta}{h}\right)^{2}, \\
& \frac{\frac{2 G M}{c^{2}}}{1-\left(\frac{m v^{2} \Theta}{h}\right)^{2}}=r .
\end{aligned}
$$

Knowing $\Theta$ and $v$, we know $r$.

We have determined $r$, and therefore $x$ of $\Phi$ on the axis. We did it by observing, as an observer situated in an almost flat space-time and not limited in his observational capacities, two parameters whose physical existence at the moment of the said observation are not questionable: The speed $v$ and the relative period $\Theta$ of the wave of our $\Phi$ particle.

We can say, again that it is entirely possible, according to General Relativity, to determine, with precision and simultaneity, the momentum and the position of the same particle.

Nota Bene: The observer is, here and in Section III, interested by the period dilated by the gravitational field of $\Omega$.

\section{CONCLUSIONS}

By applying relativistic principles to precisely and simultaneously determine the position and momentum of a massive particle, we prove in Sections III and IV the incompatibility between Heisenberg's Uncertainty Principle and time dilatation and, by extension, the incompatibility between Quantum Mechanics and General Relativity. Perhaps Einstein was right in saying that God does not play dice, perhaps the Uncertainty Principle and the theses that are based on him are correct, or perhaps none of these two models is perfectly exact. But there cannot be in one Universe these two models, precisely and simultaneously.

We can of course ask ourselves whether the observability of $E$, parameter of the particle $\Phi$, id est the physical existence of its extractible relative energy, is only a mathematical oddity, but it would be a limit to the observational capabilities of our observer, which the Heisenberg's Uncertainty Principle does not provide.

Moreover, in Section IV, the determination of the position $x$, after precise and simultaneous observation of the speed $v$ and the relative period $\Theta$ of the wave, from the point of view of an observer situated in an almost flat space-time (which are parameters whose physical existence at the moment of the observation is not questionable), confirms that it is possible to determine, by the application of relativistic principles, the speed $v$ and the position $x$ of a particle like our $\Phi$, precisely and simultaneously.

By applying relativistic principle to the point in which our nonrelativistic particle is located, we determined the position $x$ of the particle, knowing simultaneously by observation the speed $v$ of the particle. However, we get results showing their contradiction in a situation where both should 
be correct. As long as the two fundamental models which describe the functioning of our Universe were not supposed to both apply in the same situation, it was possible to conceive them as two effective approximations of the same model, integrating both the Uncertainty Principle and the relativity of space and time. But the mathematical oddity exposed seems to suggest that it is no longer possible to include both without major revision of one or the other, or both. The proposition of a theory unifying physics, of which General Relativity and Quantum Mechanics would just be approximations, now seems unlikely, the two models contradicting each other on a case in which they should both be correct. If General Relativity, proved in particular, by the detection of gravitational waves ${ }^{6,7}$ and other observations, ${ }^{8-11}$ and Quantum Mechanics, supported notably by the recent discovery of the Higgs boson, ${ }^{12,13}$ are both correct, it cannot, in such circumstances, have any theory of everything, of which the two models would just be approximations. Contemporary physics will need to review its foundations, in order to evolve in a coherent way. ${ }^{14}$

Finally, for the Uncertainty Principle not to be here refuted, we must conceive that the principle of time dilatation, in itself, may be compatible with it, and that therefore Relativity may be severely revised. The observation does not seem to show that this can be the case, the behavior of the space-time seeming to correspond too precisely to that foreseen by the General Relativity. The indeterminacy theorem does not seem to describe the physical reality. Indeed, it seems that we have just refuted the Uncertainty Principle as stated by Werner Heisenberg, by application, not contrary to the limits posed by the Uncertainty Principle itself, of relativistic principles.

It would now be interesting to consider observations validating the principles of Quantum Mechanics by revising the Uncertainty Principle or by finding an alternative.

${ }^{1}$ K. Schwarzschild, "Preußische Akademie der Wissenschaften," Sitzungsberichte 7, 189 (1916).

${ }^{2}$ W. Heisenberg, Z. Phys. 43, 172 (1927).

${ }^{3}$ E. H. Kennard, Z. Phys. 44, 326 (1927).

${ }^{4}$ H. Weyl, Gruppentheorie und Quantenmechanik (Hirzel, Leipzig, Germany, 1928).

${ }^{5}$ L. Sodickson, W. Bowman, J. Stephenson, and R. Weinstein, Phys. Rev. 124, 1851 (1961).

${ }^{6}$ LIGO Scientific Collaboration and Virgo Collaboration, Phys. Rev. Lett. 116, 241103 (2016).

${ }^{7}$ LIGO Scientific and Virgo Collaboration, Phys. Rev. Lett. 118, 221101 (2017).

${ }^{8}$ R. V. Pound and G. A. Rebka, Phys. Rev. Lett. 3, 439 (1959).

${ }^{9}$ R. V. Pound and G. A. Rebka, Phys. Rev. Lett. 4, 337 (1960).

${ }^{10}$ R. V. Pound and J. L. Snider, Phys. Rev. Lett. 13, 539 (1964).

${ }^{11}$ C. W. Chou, D. B. Hume, T. Rosenband, and D. J. Wineland, Science 329, 1630 (2010).

${ }^{12}$ ATLAS Collaboration, Phys. Lett. B 716, 1 (2012).

${ }^{13}$ CMS Collaboration, Phys. Lett. B 716, 30 (2012).

${ }^{14}$ M. V. Lokajicek, "Contemporary state of fundamental physical research," e-print arXiv: 1610.08331. 